INPLASY

PROTOCOL

To cite: Hauck et al. On Indocyanine Green

Fluorescence and

Autofluorescence in thyroid and parathyroid surgery: A systematic review. Inplasy protocol 202220067. doi: 10.37766/inplasy2022.2.0067

Received: 17 February 2022

Published: 18 February 2022

Corresponding author:

Aina Pons Talaya

ainaponstalaya8@gmail.com

Author Affiliation:

University College London

Support: Nil.

Review Stage at time of this submission: Data analysis Completed but not published.

Conflicts of interest:

None declared.

\section{On Indocyanine Green Fluorescence and Autofluorescence in thyroid and parathyroid surgery: A systematic review}

Hauck, A'; Pons, A2; Abdel-Aziz, T33.

Review question / Objective: Autofluorescence (AF) and Indocyanine Green Fluorescence (ICG) were used for the first time for parathyroid gland (PG) identification in 2011 and 2015, respectively, during thyroidectomy/parathyroidectomy. Authors reported promising results. We aim to understand the efficacy, technical challenges, cost-effectiveness, and impact on postoperative biochemical and clinical outcomes of such new techniques.

Eligibility criteria: The language filter was set to allow for publications in English, German, Spanish, and French assessing the use of ICG and/or AF for PG identification. Only titles and abstracts, followed by the full text dating from 2008 to 2020 have been considered in this review. Existing systematic reviews were excluded from the results.

INPLASY registration number: This protocol was registered with the International Platform of Registered Systematic Review and Meta-Analysis Protocols (INPLASY) on 18 February 2022 and was last updated on 18 February 2022 (registration number INPLASY202220067).

\section{INTRODUCTION}

Review question / Objective: Autofluorescence (AF) and Indocyanine Green Fluorescence (ICG) were used for the first time for parathyroid gland (PG) identification in 2011 and 2015 , respectively, during thyroidectomy/ parathyroidectomy. Authors reported promising results. We aim to understand the efficacy, technical challenges, costeffectiveness, and impact on postoperative 
biochemical and clinical outcomes of such new techniques.

Condition being studied: Different techniques to identify PG intraoperatively have been developed in the past decades. Methylene blue was first used intraoperatively in the $70 \mathrm{~s}$, but concerns regarding neurotoxicity were expressed. 5ALA can also be used for identification of the PG9, but patients need to be protected from sunlight for 24-48h to prevent phototoxicity and photobleaching. Intraoperative parathyroid hormone (IOPTH) monitoring was introduced in 1988 by Nussbaum et al. and has been found to consistently outperform preoperative imaging such as ultrasound and nuclear scan MIBI in predicting cure. Autofluorescence (AF) for PG identification - based on the fluorescence emitted by the PG when exposed to a near infrared, 750-800 $\mathrm{nm}$ wavelength - was discovered in 2008 and was used for the first time in 2011. Since then, clinical devices have been approved by the FDA for its use intraoperatively. Similarly, indocyanine green fluorescence imaging (ICG) is injected intravenously and binds to plasma lipoproteins. This dye can thereafter be excited by near infrared wavelengths, resulting in fluorescence and facilitating the PG identification. Since 2015, ICG has been reported as an advantageous technique that can safely be used in medical practice.

\section{METHODS}

Search strategy: This study was conducted according to PRISMA guidelines21. A systematic search of PubMed/MEDLINE has been performed with the help of MeSH: ((Thyroidectomy[Title/Abstract]) OR (Parathyroidectomy[Title/Abstract]) OR ((thyroid[MeSH Major Topic]) AND (surgery[MeSH Subheading])) OR ((parathyroid[MeSH Major Topic]) AND (surgery[MeSH Subheading]))) AND ((Nearinfrared [ Title/ Abstract]) OR (Fluorescence[Title/Abstract]) OR (ICG[Title/Abstract]) OR (indocyanine[Title/ Abstract])). The filter on publication date was set to 2008-2020. The search results list was downloaded on 07.08.2020. The language filter was set to allow for publications in English, German, Spanish, and French assessing the use of ICG and/ or AF for PG identification. Only titles and abstracts, followed by the full text dating from 2008 to 2020 have been considered in this review. Existing systematic reviews were excluded from the results. Data was extracted from text, tables, and figures by two authors independently and summarised in the form of a shared table. When unclear, inclusion or exclusion of the paper was discussed between the authors and a joint decision was reached. Data included in the table was double-checked posteriorly by the two authors independently.

Participant or population: Patients undergoing thyroidectomy and/or parathyroidectomy.

Intervention: Patients undergoing thyroidectomy and/or parathyroidectomy.

Comparator: Traditional methods for PG identification.

Study designs to be included: 3 RCT, 33 observational studies and 22 case series or reports.

Eligibility criteria: The language filter was set to allow for publications in English, German, Spanish, and French assessing the use of ICG and/or AF for PG identification. Only titles and abstracts, followed by the full text dating from 2008 to 2020 have been considered in this review. Existing systematic reviews were excluded from the results.

\section{Information sources: PubMed/MEDLINE}

Main outcome(s): Outcomes (biochemical, clinical, need for long term treatment, length of stay, reoperation).

Additional outcome(s): Identification of parathyroid glands, other structures displaying ICG/AF, factors influencing the ICG/AF signal, distinction between pathological and healthy Viability, influence 
on surgeon's choice, number of parathyroid glands found in specimen Need to turn off lights, duration of surgery, undesirable events, cost effectiveness.

Data management: No patient identification.

Quality assessment / Risk of bias analysis: One of the limitations of our study is that "autofluorescence" is not considered as a MeSH term. We used synonyms for the literature selection process, but this could have led to a less precise search process. Another limitation is that the cohorts where not big enough to allow subgroup analysis. The applications of ICG and AF could differ in different subgroups of patients and its efficacy could vary for PG identification as the characteristics of disease progression and response to surgery might between subgroups.

Strategy of data synthesis: This study was conducted according to PRISMA guidelines21. A systematic search of PubMed/MEDLINE has been performed with the help of MeSH: ((Thyroidectomy[Title/Abstract]) OR (Parathyroidectomy[Title/Abstract]) OR ((thyroid[MeSH Major Topic]) AND (surgery[MeSH Subheading])) OR ((parathyroid[MeSH Major Topic]) AND (surgery[MeSH Subheading]))) AND ((Nearinfrared [ Title/Abstract]) OR (Fluorescence[Title/Abstract]) OR (ICG[Title/Abstract]) OR (indocyanine[Title/ Abstract])). The filter on publication date was set to 2008-2020. The search results list was downloaded on 07.08.2020. The language filter was set to allow for publications in English, German, Spanish, and French assessing the use of ICG and/ or AF for PG identification. Only titles and abstracts, followed by the full text dating from 2008 to 2020 have been considered in this review. Existing systematic reviews were excluded from the results. Data was extracted from text, tables, and figures by two authors independently and summarised in the form of a shared table. When unclear, inclusion or exclusion of the paper was discussed between the authors and a joint decision was reached. Data included in the table was double-checked posteriorly by the two authors independently.

Subgroup analysis: Data was extracted from text, tables, and figures by two authors independently and summarised in the form of a shared table.

Sensitivity analysis: No sensitivity analysis.

Language: English, German, Spanish, and French.

Country(ies) involved: United Kingdom.

Keywords: ICG, autofluorescence, thyroid, parathyroid, surgery.

Contributions of each author:

Author 1 - Aina Pons - Conception, scoping, data analysis, summarising of data, writing of manuscript.

Email: ainapons8@gmail.com

Author 2 - Annalisa Hauck - Conception, scoping, data analysis, summarising of data, writing of manuscript.

Email: annalisa.hauck@gmail.com

Author 3 - Tarek Abdel Aziz - Conception, scoping, data analysis, summarising of data, writing of manuscript, supervision.

Email: tarek.abdel-aziz@nhs.net 